Vorlesungen über Darstellende Geometrie. Von Dr. F. v. Dalwigk, Privatdozent in Marburg. II. Band: Perspektive, Zentralkollineation und Grundzüge der Photogrammmetrie. Über 130 Fig., 322 S. Verlag B. G. Teubner, 1914. Preis geb. 11 M.-.

Im ersten Teil behandelt der Verfasser die malerische Perspektive; hiebei bespricht er sehr ansführlich die Darstellang und Schattenkonstruktion eines Hauses, später, nachdem er die Darstellung des Kreises erledigt hat, verschiedene architektonische Aufgaben über Rundbogen, Säulenhallen usw. Im zweiten Teil geht er zur freien Perspektive über, deren Elemente schon im ersten Teil gelegentlich eingeflochten wurden, wendet sich sodann der Zentralkollineation und der Reliefperspektive zu. In einem Anhange bespricht er, allerdings nur auf einigen Seiten, die Grundzüge der Photogrammetrie und schließt nach ]zurzen ergänzenden Bemerkungen über die malerische Perspektive mit einigen historischen Angaben.

Es ist an diesem Buche nichts Wesentliches auszusetzen (daß der Verfasser sich manchmal etwas kürzer hätte ausdrücken können, ohne die Klarheit zu beeinträchtigen, $\mathrm{da} \beta$ an einigen Stellen Vereinfachungen möglich wären, sei nebenbei bemerkt); anderseits ist aber auch nichts Besonderes hervorzaheben und muß gesagt werden, daß sich in dem Buche keine neuen Gesichtspunkte vorfinden. Jedenfalls ist es mit großem Fleiß and mit Sorgfalt gearbeitet und wird seinen Zweck, denen, die an Universitäten angewandte Mathematik studieren, eine leichtfaßliche Einführung in die darstellende Geometrie zu bieten, erreichen.

o. Danzer.

\title{
Über die Dirichletsche Theorie der Fourierschen Reihen.
} Von C. Neamann. (Abhdl. der math. phys. Kl. d. kgl. sächs. Ges. der Wiss., Bd. XXXIII, Nr. I.) B. G. Teubner, Leipzig, 1914. 80 S. 3 M.-.

Der Verfasser zeigt in dieser Arbeit in der ihm eigenen breiten Darstellungsweise, daß eine mit Ausnahme ron endlich vielen Punkten $\alpha$ abteilungsweise stetige Funktion, die mit Ausnahme von endlich vielen Strecken $\gamma \delta$ abteilungsweise monoton ist, eine Fourier-Reihe besitzt, die in jedem Stetigkeitsintervalle; das von den Strecken $\gamma \delta$ und den Punkten $\alpha$ durch irgend welche Zwischenräume getrennt ist, gleichmäßig konvergiert. Dabei ist vorausgesetzt, daß jeder Punkt $\alpha$ ein Dirichletscher Punkt ist, d. h. daß er außerhalb der Strecken $\gamma \delta$ liegt und daß man über ibn hinwegintegrieren kann.

Algebraische Kurven. I. Gestaltliche Verhältnisse. Von H. Wiel eitner. (Slg. Göschen, Bdch. 435.) Göschen, Leipzig, 1914. 146 S. 90 Pf.

Das vorliegende Bändchen bietet eine mit vielen Beispielen ausgestattete Einführung in die Theorie der algebraischen Kurven, wobei im wesentlichen nur die Kurven 3. u. 4. Ordnung, bezw. 3. u. 4. Klasse eingehender besprochen werden. Auf Seite 8 hat es zu heißen: nach Ersetzung von $x$ durch $\rho y$ ( $p$ willkürliche Konstante). 